\title{
CONSIDERACIONES SOBRE ALGUNOS ERRORES CONCEPTUALES EN EL APRENDIZAJE DE LA QUIMICA EN EL BACHILLERATO
}

\author{
CAMAÑo, A., MAYOS, C., MAESTRE, G, y VENTURA, T. \\ del GRUP RECERCA \\ Comunicación presentada en las Primeras Jornadas de \\ Investigación Didáctica de la Física y la Química. \\ Valencia 1982.
}

\section{SUMMARY}

Some mistakes frequently found in the learning process of Chemistry in secondary schools are dealth with. Specifically:

- the difficulty in making the difference between mixed and compound substance.

- the difficulty in reasoning the discontinuous nature of matter,

- the associations atom-element and molecule-compound,

- the non comprenhension of the mol concept, etc...

Some suggested solutions are also included.

\section{INTRODUCCION}

El objetivo de este artículo es presentar una serie de consideraciones sobre algunos errores que se dan frecuentemente en el aprendizaje de la química en el bachillerato, que creemos son consecuencia de la utilización de una didáctica ajena a la metodología científica y a la evolución histórica de los conceptos.

Los ejemplos que se aportan corresponden a las propuestas didácticas contenidas en el Proyecto Faraday del Grup Recerca (1980-82) y son fruto de la experiencia obtenida a lo largo de cuatro años de su utilización.

\section{LA DIFICULTAD DE DIFERENCIAR ENTRE MEZCLA Y COMPUESTO QUTMUCO}

Muchos alumnos no distinguen claramente las mezclas de los compuestos, puesto que en ambos casos intervienen varios componentes.

El origen de este error creemos que proviene de los hechos siguientes:

- No se ha llegado a adquirir los conceptos operacionales de mezcla, sustancia pura, elemento y compuesto, que permitan distinguir entre la separación de los componentes de una mezcla y la separación de los elementos de un compuesto (descomposición).
- Se han introducido de forma precipitada las de. finiciones conceptuales que proporciona la teoría atómica. Una primera definición de compuesto como aquella sustancia constituida por átomos distintos puede aplicarse también a las mezclas. El problema de distinción resulta entonces insoluble si no se introduce a la vez el concepto de enlace químico, lo que complicaría todavia más la situación.

Nuestra propuesta es la utilización experimental de métodos de separación físicos, tales como la destilación, la cristalización, la sublimación, etc., que permita a los alumnos llegar a los conceptos operacionales de mezcla y sustancia pura, del mismo modo que el calentamiento enérgico y la electrólisis permite distinguir, posteriomente, entre sustancias puras simples (elementos) y compuestas (compuestos).

La distinción entre mezclas y compuestos se puede realizar en el marco operacional citado a través del conjunto de los siguientes hechos:

- Un compuesto es una sustancia pura y como tal su temperatura de fusión y de ebullición se mantiene constante, cosa que no ocurre en una mezcla o disolución (debido a que en el cambio de estado varía su composición). 
- En una mezcla las propiedades dependen de las cantidades de los componentes que constituyen la misma. En un compuesto las propiedades son fijas.

- Se necesitan condiciones más drásticas cuando se trata de separar los elementos de un compuesto que en el caso de separar los componentes de una mezcla.

- En los compuestos las cantidades de los elementos que se combinan guardan siempre la misma relación.

\section{LA NECESWAD DE JUSTIFICAR LA NATU- RALEZA DISCONTINUA DE LA MATERIA}

En el desarrollo de una teoría de la materia que explique sus propiedades y las diferencias observadas entre mezclas, compuestos y elementos, nos encontramos que existe ya en los alumnos una aceptación previa acrítica de su carácter discontinuo. Estamos pues ante un caso, en el que no hay que derribar un concepto previo erróneo sino fundamentar críticamente tal concepto.

Una solución al problema es presentar la hipótesis de la continuidad de la materia como plausible para que de este modo la pregunta: ¿por qué creemos en la existencia de los átomos?, adquiera todo su significado.

Aparece entonces la cuestión de valorar qué pruebas son realmente relevantes respecto al carácter discontinuo de la materia, cuáles lo sugieren con una cierta probabilidad y cuáles en realidad no aportan evidencia alguna, a pesar de ser frecuentemente citadas (Wright 1981). Entre las primeras cabe referirse a la difusión de los gases y de los sólidos en líquidos y a las leyes de las proporciones definidas y de las proporciones múltiples; entre las segundas, al crecimiento de los cristales y la evaporación; y entre las últimas, a la contracción de volumen al mezclar dos líquidos y la persistencia del color al diluir una disolución coloreada.

\section{ATOMO-ELEMENTO Y MOLECULA- COMPUESTO: UNA ASOCLACION CONCEP. TUAL QUE DEBE EVTTARSE}

Un problema que se plantea en una aproximación histórica a la idea de átomo y molécula a través de la teoría atómica de Dalton, es la de asociar, tal como ocurría en la época de Dalton, los conceptos de átomo con elemento y de molécula con compuesto. El alumno es inducido, de esta forma, a pensar que un elemento está formado siempre por átomos (de ahí la resistencia posterior a asimilar que gases como el hidrógeno son moleculares) y que un compuesto está formado siempre por moléculas (de ahi el error de tomar el $\mathrm{NaCl}$ y el $\mathrm{SiO}_{2}$ como moleculares).

La solución de este problema requiere:

- La introducción del concepto de estructura giv gante a la vez que el de molécula (Nuffield 1972). Se entiende por estructura gigante un conjunto ininterrumpido de átomos de la misma o diferente clase, combinados entre sí de forma que no pueden diferenciarse unidades discretas. La estructura gigante es pues un concepto globalizador de los sólidos metálicos, iónicos y reticulares y puede ser utilizado previamente a la clasificación de los sólidos.

- Una aproximación al concepto de fórmula quimica que deje bien claro que el conocimiento de una fómula (empirica) no implica conocimiento alguno sobre la estructura de la sustancia.

\section{SOBRE EL CONCEPTO DE MOL,}

Mucha es la literatura escrita sobre las dificultades que entraña la comprensión del concepto de mol y las ventajas $\mathrm{e}$ inconvenientes de sus diferentes for mas de presentación.

La definición de mol como cantidad de sustancia que contiene $6,02 \times 10^{23}$ entidades específicas (átomos, moléculas, etc.) siendo evidentemente clara y concisa, deja la incógnita del número $6,02 \times 10^{23}$. Si el mol es simplemente la "docena" del químico para contar las unidades materiales que forman una porción de materia, entonces cabe preguntarse ¿por que no haber escogido, por ejemplo, $10^{23}$ ?

Un enfoque que intente dar respuesta a esta dificultad debe partir del origen histórico del concepto de mol, haciendo ver el problema al cual respon día: disponer de cantidades de diferentes sustancias que contuvieran el mismo número de átomos o moléculas. La solución fue tomar cantidades en gramos iguales a las masas atómicas relativas.

El problema de este enfoque es entonces el que el alumno asocie el concepto de mol al de masa molar. Por ejemplo, asocie $12 \mathrm{~g}$ de carbono con $1 \mathrm{~mol}$ de átomos de carbono y no con la masa de $1 \mathrm{~mol}$ de átomos de carbono.

Una introducción del mol que salve al máximo este posible error conceptual y por otro lado explique el valor numérico de la constante de Avogadro, aparentemente arbitraria, pensamos requiere 
la utilización consecutiva de ambas aproximaciones: introducción histórica del mol y, a continuación, su definición actual en el SI.

\section{POR UNA UTLLZACION DE UN LENGUAJE QUIMICO QUE NO MPLIQUE ERRORES CONCEPTUALES}

En los últimos años se ha destacado el papel importante que juega el lenguaje en la enseñanza de las ciencias, no únicamente como medio de comunicación de las ideas, sino como una manera de interpretar y dar sentido a las experiencias. Podemos decir que en cierta forma "comprender una teoria es aprender un lenguajen.

En este sentido la utilización de la nomenclatura recomendada por la IUPAC para designar las magnitudes químicas, no solo evita ambigüedades sino que permite en muchos casos precisar el significado conceptual de éstas (Caamaño 1982). Citemos algunos ejemplos referidos a magnitudes moleculares:
- La forma correcta de expresar la cantidad de sustancia evita la confusión conceptual que se generaba al designar como átomo-gramo a un mol de moléculas.

- Las nomenclaturas y símbolos diferenciados para la masa atómica o molecular relativa, la masa atómica y la masa molar favorece la distinción entre estas magnitudes.

- Las actuales denominaciones de constante de Avogadro y constante de Faraday evitan confundir estas magnitudes con un número puro y con una unidad de electricidad, respectivamente.

Sin embargo, a pesar del esfuerzo de utilizar un lenguaje preciso, el significado de algunos conceptos en el lenguaje corriente nos induce a veces a confusión. Por ejemplo, la palabra cantidad remite sin desearlo a entender masa, cuando en el len. guaje químico significa cantidad de sustancia. La solución habitual de referimos a esta magnitud como número de moles tampoco es satisfactoria puesto que la cantidad de sustancia es una magnitud fisica con dimensiones y no un número.

\section{REFERENCLAS BIBLIOGRAFICAS}

GRUP RECERCA, 1980-82, Proyecto Faraday (I. Ouímica para el bachillerato) Guia general. Unidades didácticas (gúa del alumno y del profesor), ICE de la Universidad Autó. noma de Barceiona: Bellaterra, Barcelona.

WRIGHT, P.G., 1981, Evidence and non-evidence for the existence of atoms and molecules, Education in Chemistry, 18. 3, p. 74.
NUFFIELD, 1972, Quimica. Manuel pora profesores, cap. 4, Reverté.

CAAMAN̈O, A., 1982, Las nuevas tendencias en la expresión y nomenclatura de las magnitudes fisicas y químicas y de sus unidades, Jornadas de Didáctica de la Física y Quínica, ICE de la Universidad de Valencia. 1982. 